(1)

CrossMark

\title{
Cyclosporine: an old weapon in the fight against coronaviruses
}

\author{
Adam Molyvdas and Sadis Matalon
}

Affiliation: Depts of Anesthesiology and Perioperative Medicine, Division of Translational and Molecular Biomedicine and Pulmonary Injury and Repair Center, University of Alabama at Birmingham, School of Medicine, Birmingham, AL, USA.

Correspondence: Sadis Matalon, BMR II 224, 901 19th Street South, Birmingham, AL 35205-3703, USA. E-mail: smatalonduabmc.edu

@ERSpublications

Cyclosporine inhibits the replication of coronaviruses and could potentially suppress the cytokine storm associated with coronavirus infections https://bit.ly/39x2PSt

Cite this article as: Molyvdas A, Matalon S. Cyclosporine: an old weapon in the fight against coronaviruses. Eur Respir J 2020; 56: 2002484 [https://doi.org/10.1183/13993003.02484-2020].

Coronaviruses have been known to cause respiratory infections in humans and intestinal infections in other mammals. Severe acute respiratory syndrome coronavirus 2 (SARS-CoV-2), which causes coronavirus disease 2019 (COVID-19), is the seventh virus of the Coronaviridae family that is known to infect humans. Until 2002, four Coronaviruses infecting humans were described (HCoV-NL63, HCoV-229E, HCoV-OC43 and HKU1). These viruses caused only mild respiratory diseases in immunocompetent hosts. Since 2002, three highly pathogenic viruses from this family have been identified. SARS-CoV (also referred to as SARS-CoV-1) is an enveloped, positive-sense, single-stranded RNA virus which infects the epithelial cells within the lungs. The virus enters the host cell by binding to angiotensin-converting enzyme 2 (ACE2) [1]. It infects humans, bats and palm civets [1]. It emerged in 2002-2003 with an approximate 10\% mortality but limited transmissibility (approximately 8100 cases, $\mathrm{R}_{0}<1$ ) [2-5]. Infected persons develop influenza-like symptoms which may progress to pneumonia, acute respiratory distress syndrome (ARDS) and death from respiratory failure and multi-organ failure. Middle East respiratory syndrome coronavirus (MERS-CoV) emerged in 2012 in Saudi Arabia and was the second highly pathogenic virus of this family [6], yet with relatively low rate of transmission. MERS-CoV had even higher mortality than SARS-CoV (30\%), but again was characterised by low transmissibility (approximately 2500 cases since 2012, $\mathrm{R}_{0}<1$ ) [6]. Typical MERS symptoms include fever, cough and shortness of breath. Pneumonia is common, but not always present. Gastrointestinal symptoms, including diarrhoea, have also been reported. Some laboratory-confirmed cases of MERS-CoV infection are reported as asymptomatic. Most of these asymptomatic cases have been detected following aggressive contact tracing of a laboratory-confirmed case. Dromedary camels are a major reservoir host for this virus.

SARS-CoV-2 emerged in 2019 in Wuhan, China and is characterised by a much higher person-to-person transmissibility than SARS-CoV or MERS-CoV. The SARS-CoV-2 virus, responsible for COVID-19 disease, contains four main structural proteins. One of them, the $S$ (spike) glycoprotein, a transmembrane protein, found in the outer portion of the virus, forms homotrimers binding with human ACE2 receptors of host cells [7]. The SARS CoV-2 S protein has significant homology with the SARS-CoV-1 equivalent protein [8], which also binds to ACE2 receptors [9]. However, the SARS CoV-2 S protein binds with higher affinity to ACE2 receptors [10] due to the presence of a furin-like cleavage site $\left({ }^{682} \mathrm{RRAR} / \mathrm{S}^{686}\right)$ inserted in the S1/S2 protease cleavage site [11]. A considerable percentage of COVID-19 patients (5-19\%) 
develop ARDS within 24-48 h after onset of symptoms, requiring mechanical ventilation with supplemental oxygen and succumb to the disease. The mortality of patients who develop ARDS is $49 \%$ [12]. Many patients with COVID-19 develop multi-organ failure. The leading causes of death are ARDS, septic shock with multi-organ failure, haemorrhage/coagulopathy (disseminated intravascular coagulopathy), acute heart/liver/kidney injury, and secondary bacterial infections [12]. The mean length of stay in the ICU is about 8 days for severely ill patients with considerable variation.

Most infected individuals are asymptomatic and may spread the disease by respiratory droplets carrying the viruses. Persons over 60 years old with a variety of health ailments such as diabetes, hypertension, chronic obstructive lung disease, asthma or compromised kidney function are highly susceptible to SARS CoV-2 and develop a more severe disease when infected. It has been proposed that a common factor in all comorbidities is the presence of high levels of plasmin in the circulation which cleaves the furin site of the virus, increasing its infectivity [12]. Although a number of clinical trials are ongoing (approximately 1300 interventional trials approved worldwide when this editorial was written, according to the NIH database [13]) no effective treatments are available and patients receive mainly supportive treatment.

MERS-CoV and SARS-CoV pathogenesis has been studied more extensively than SARS-CoV-2. MERS-CoV and SARS-CoV disease evolves through two different mechanisms: immunopathology and viral evasion [1]. SARS-CoV and MERS-CoV have been shown to induce a cytokine storm that results in a positive feedback loop of immune system activation that becomes independent of viral load [14-16]. Transfection of human cells with plasmids containing either the S or N proteins of SARS CoV-1 activate various isoforms of $\mathrm{PKC}$ and decrease the activity of the amiloride sensitive epithelial sodium channel $(\mathrm{ENaC})[17]$, which may impede the clearance of alveolar oedema and worsen the outcome of the disease [18]. Furthermore, SARS-CoV and MERS-CoV utilise a wide variety of mechanisms to evade host immune detection [1]. SARS-CoV and MERS-CoV replicate in vesicles that lack pattern recognition receptors (PPRs) $[19,20]$. In addition, SARS-CoV and MERS-CoV produce several proteins that interfere with signalling cascades downstream of PPRs [1]. Finally, there is evidence that SARS-CoV and MERS-CoV can inhibit the activation of type I interferons through SARS-CoV PLpro and MERS-CoV PLpro respectively [20-23]. SARS-CoV-2 has been hypothesised to work in a similar manner due to its close relationship with SARS-CoV and MERS-CoV.

The paper by SAUERHERING et al. [24] attempts not only to provide a possible therapeutic treatment for MERS-CoV, but also provides valuable insights on the mechanism of action of their proposed treatment, namely cyclosporin A (CsA).

CsA was isolated from the fungus Tolypocladium inflatum in 1971 and came into medical use in 1983. Currently, CsA is extensively prescribed in the USA and most of the western world. In the USA, CsA is approved by the Food and Drug Administration to treat and prevent graft-versus-host disease in bone marrow transplantation and to prevent rejection of kidney, heart and liver transplants. Furthermore, CsA is approved for the treatment of rheumatoid arthritis, as well as other autoimmune-related disorders.

CsA has been shown to inhibit in vitro the replication of several coronaviruses, including SARS-CoV and MERS-CoV (figure 1a) [25-27]. Under normal conditions after T-cell receptor activation, cyclophilin A (Cyp-A) and calcineurin form a complex that leads to the activation of the nuclear factor of activated T-cell (NF-AT) [28, 29]. CsA exerts its immunosuppressive effects through the binding of Cyp-A and calcineurin preventing the activation of NF-AT (figure 1b) [28-30]. CsA binds Cyp-A at its hydrophobic pocket inhibiting its isomerase function, but the immunosuppressive properties of CsA are independent of this function [31, 32]. The CsA-Cyp-A complex binds calcineurin (an important downstream mediator of T-cell receptors) without activating it [33], blocking downstream signalling of the T-cell receptor. Furthermore, the CsA-Cyp-A complex has been shown to be essential to the suppression of viral replication in several viruses and SAUERHERING et al. [24] provide insights about the mechanism involved in this function of the complex [34]. Cyp-A has similar structure to other cyclophilins and CsA does form complexes with them and affects different mechanisms and cell functions. An important example is the binding to Cyp-D. CsA binds to Cyp-D, preventing cell death under stress conditions by inhibiting the opening of the mitochondrial permeability transition pore (mPTP), a pathophysiological event triggered under stress conditions (figure 1c) [28].

The non-structural protein 1 of SARS-CoV has been found to induce IL-2 production through NF-AT [35, 36]. CsA has been shown to significantly inhibit IFN- $\gamma$, IL-4, and IL-17 production from memory T-cells in vitro within $24 \mathrm{~h}$ of administration [37]. These compounds also inhibited T-cell differentiation into the Th1, Th2 and Th17 subsets, even when used at a low concentration within a 6-day in vitro protocol [37].

The authors for the first time show that CsA treatment not only inhibits MERS-CoV viral replication in vitro but also in a murine in vivo model. Furthermore, the in vivo model utilised showed improved 


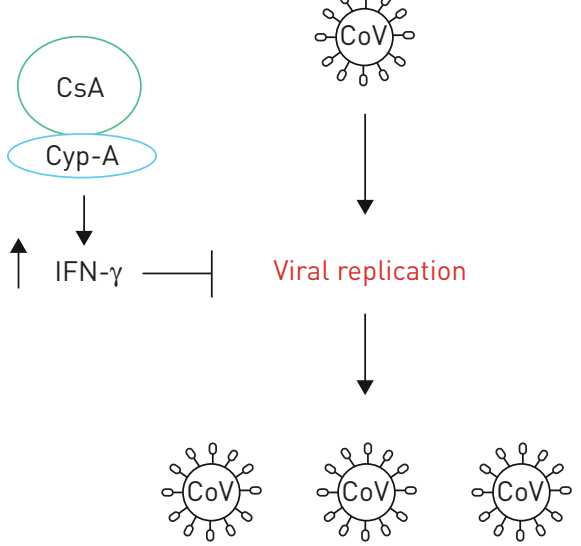

b)<smiles></smiles>

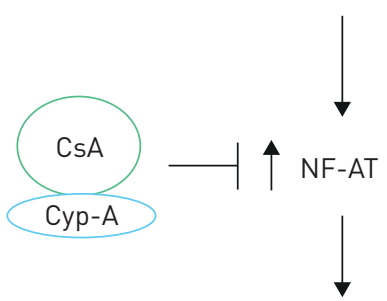

c)

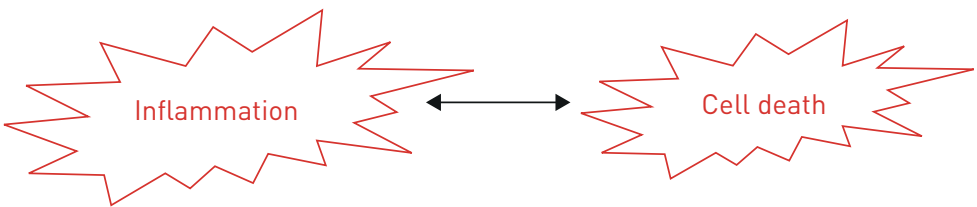

FIGURE 1 Schematic overview of the interactions of cyclosporine A (CsA) and coronaviruses. a) CsA binds to cyclophilin A (Cyp-A) and upregulates interferon- $\lambda$ (IFN $\lambda$ ) which blocks viral replication. b) Coronaviruses activate the nuclear factor of activated T-cell (NF-AT), which triggers the release of inflammatory cytokines and causes inflammation. The CsA-Cyp-A complex prevents the activation NF-AT reducing inflammation. cl Coronaviruses cause aberrant opening of the mitochondrial permeability transition pore (mPTP), which results in cell death. CsA in complex with cyclophilin-D (Cyp-D) prevents the opening of MPTP reducing cell damage and cell death.

disease outcomes. These results by themselves are extremely important. To our knowledge this the first in vivo study showing inhibition of viral replication for any of the highly pathogenic coronaviruses. This finding is important as it provides experimental evidence that cyclophilin inhibitors and CsA in particular are effective not only in the isolated setting of an in vitro setting but also in the complicated setting of a whole animal. The successful mitigation of lung pathology presented after CsA treatment indicates the potential therapeutic usages of CsA against MERS-CoV and potentially other coronaviruses.

The authors also provided important insights on the mechanism of action of CsA. They showed that the activation of the interferon regulatory factor 1-interferon lamda (IRF1-IFN $\lambda$ ) signalling pathway is essential to the MERS-CoV antiviral effects of CsA. The IRF1-IFN $\lambda$ signalling axis is an extensively studied pathway that has been shown to be involved in antiviral mechanisms, most notably in influenza host defence, and malfunction of this pathway is implicated in the progression of viral infections to chronicity $[38,39]$. Using an appropriate combination of in vivo and in vitro experiments the authors showed that CsA upregulates the IRF1-IFN $\lambda$ signalling pathway in vivo. In vitro they showed that inhibition of the IRF1-IFN $\lambda$ signalling pathway blocked the antiviral properties of CsA and allowed viral replication.

Moving forward it would be interesting to investigate whether the improved in vivo outcomes of this study are not only due to the antiviral effects that the authors investigate thoroughly, but also to a downregulation of the cytokine storm that is evident in coronavirus infections. Possible tools in this research are the transgenic murine models (Ifnlr1 $1^{-/}$and Ifnar $1^{-/}$) currently utilised in influenza research [38]. CsA is an appropriate countermeasure against cytokine storm in a number of diseases and in particular secondary haemophagocytic lymphohistiocytosis [40-42]. Furthermore, determination of the timing of the administration of this agent is important. Early administration may render the host susceptible to bacterial infections, which are known to enhance mortality significantly. Administration later on in the disease, may limit the onset of the cytokine storm which contributes to mortality from coronavirus infections. Namely, how early during the infection and before or during the "cytokine storm" CsA should be applied.

Before considering CsA for human clinical trials to treat COVID-19, a careful evaluation of cost/benefit should take place given the variety of side-effects including increased susceptibility to infections, nausea, vomiting and triggering of cancer development among others [43]. Furthermore, aerosolised administration of CsA has been proposed as a treatment or potential preventative measure of bronchiolitis obliterans syndrome in lung transplant patients $[44,45]$. The results of these clinical trials have provided interesting insights and have shown that CsA, when administered as an aerosol, has little or no systemic toxicity. Considering the acute life-threating aspects of COVID-19 from respiratory complications of the disease, a direct local application could make sense. However, this approach has a potential pitfall. The virus has several reservoirs outside the pulmonary system. A local application of CsA might not effectively combat those virus reservoirs, leading to unforeseen complications. It would also be useful to identify 
more specific targets and therapeutic agents targeting the IRF1-IFN $\lambda$ signalling pathway. This is more possible now thanks to the very important work on the mechanism proposed by SAUERHERING et al. [24].

Considering the familial relationship between MERS-CoV, SARS-CoV and SARS-CoV-2, this study is extremely relevant during the current COVID-19 pandemic. The authors provide useful insights to the mechanism of action and possible therapeutic role of CsA against coronaviruses. Although CsA is not currently approved for use in SARS-CoV-2 cases, the results of this study warrant a more careful investigation and preclinical studies into the possible use of CsA as a therapeutic agent against COVID-19.

Support statement: This work was supported by the CounterACT Program, National Institutes of Health Office of the Director (NIH OD), the National Institute of Neurological Disorders and Stroke (NINDS), and the National Institute of Environmental Health Sciences (NIEHS) (grant numbers 5UO1 ES026458; 3UO1 ES026458 03S1; 5UO1 ES027697 to S. Matalon). Funding information for this article has been deposited with the Crossref Funder Registry.

Conflict of interest: A. Molyvdas has nothing to disclose. S. Matalon has nothing to disclose.

\section{References}

1 De Wit E, van Doremalen N, Falzarano D, et al. SARS and MERS: recent insights into emerging coronaviruses. Nat Rev Microbiol 2016; 14: 523-534.

2 Drosten C, Günther S, Preiser W, et al. Identification of a novel coronavirus in patients with severe acute respiratory syndrome. N Engl J Med 2003; 348: 1967-1976.

3 Ksiazek TG, Erdman D, Goldsmith CS, et al. A novel coronavirus associated with severe acute respiratory syndrome. N Engl J Med 2003; 348: 1953-1966.

4 Peiris JS, Lai ST, Poon LL, et al. Coronavirus as a possible cause of severe acute respiratory syndrome. Lancet 2003; 361: 1319-1325.

5 World Health Organization. Summary of Probable SARS Cases with Onset of Illness from 1 November 2002 to 31 July 2003. www.who.int/csr/sars/country/table2004_04_21/en/

6 World Health Organization. Middle East Respiratory Syndrome Coronavirus (MERS-CoV). www.who.int/ emergencies/mers-cov/en/

7 Hoffmann M, Kleine-Weber H, Schroeder S, et al. SARS-CoV-2 cell entry depends on ACE2 and TMPRSS2 and is blocked by a clinically proven protease inhibitor. Cell 2020; 181: 271-280.

8 Ou X, Liu Y, Lei X, et al. Characterization of spike glycoprotein of SARS-CoV-2 on virus entry and its immune cross-reactivity with SARS-CoV. Nat Commun 2020; 11: 1620.

9 Kuba K, Imai Y, Rao S, et al. A crucial role of angiotensin converting enzyme 2 (ACE2) in SARS coronavirus-induced lung injury. Nat Med 2005; 11: 875-879.

10 Wrapp D, Wang N, Corbett KS, et al. Cryo-EM structure of the 2019-nCoV spike in the prefusion conformation. Science 2020; 367: 1260-1263.

11 Coutard B, Valle C, de Lamballerie X, et al. The spike glycoprotein of the new coronavirus 2019-nCoV contains a furin-like cleavage site absent in CoV of the same clade. Antiviral Res 2020; 176: 104742.

12 Ji H-L, Zhao R, Matalon S, et al. Elevated plasmin(ogen) as a common risk factor for COVID-19 susceptibility. Physiol Rev 2020; 100: 1065-1075.

13 US National Library of Medicine. Clinicaltrials.gov. https://clinicaltrials.gov/ct2/results?cond=Covid19\&Search= Apply\&recrs $=$ b\&recrs $=a \&$ recrs $=$ f\&recrs $=d \&$ age_v $=\& g n d r=\&$ type $=I n t r \& r s l t=$

14 Drosten C, Seilmaier M, Corman VM, et al. Clinical features and virological analysis of a case of Middle East respiratory syndrome coronavirus infection. Lancet Infect Dis 2013; 13: 745-751.

15 Poissy J, Goffard A, Parmentier-Decrucq E, et al. Kinetics and pattern of viral excretion in biological specimens of two MERS-CoV cases. J Clin Virol 2014; 61: 275-278.

16 Wang WK, Chen SY, Liu IJ, et al. Temporal relationship of viral load, ribavirin, interleukin (IL)-6, IL-8, and clinical progression in patients with severe acute respiratory syndrome. Clin Infect Dis 2004; 39: 1071-1075.

17 Ji HL, Song W, Gao Z, et al. SARS-CoV proteins decrease levels and activity of human ENaC via activation of distinct PKC isoforms. Am J Physiol Lung Cell Mol Physiol 2009; 296: L372-L383.

18 Matthay MA, Wiener-Kronish JP. Intact epithelial barrier function is critical for the resolution of alveolar edema in humans. Am Rev Respir Dis 1990; 142: 1250-1257.

19 de Wilde AH, Raj VS, Oudshoorn D, et al. MERS-coronavirus replication induces severe in vitro cytopathology and is strongly inhibited by cyclosporin A or interferon-alpha treatment. J Gen Virol 2013; 94: 1749-1760.

20 Snijder EJ, van der Meer Y, Zevenhoven-Dobbe J, et al. Ultrastructure and origin of membrane vesicles associated with the severe acute respiratory syndrome coronavirus replication complex. J Virol 2006; 80: 5927-5940.

21 Bailey-Elkin BA, Knaap RC, Johnson GG, et al. Crystal structure of the Middle East respiratory syndrome coronavirus (MERS-CoV) papain-like protease bound to ubiquitin facilitates targeted disruption of deubiquitinating activity to demonstrate its role in innate immune suppression. J Biol Chem 2014; 289: 34667-34682.

22 Frieman M, Ratia K, Johnston RE, et al. Severe acute respiratory syndrome coronavirus papain-like protease ubiquitin-like domain and catalytic domain regulate antagonism of IRF3 and NF-kappaB signaling. $J$ Virol 2009; 83: 6689-6705.

23 Matthews K, Schäfer A, Pham A, et al. The SARS coronavirus papain like protease can inhibit IRF3 at a post activation step that requires deubiquitination activity. Virol J 2014; 11: 209.

24 Sauerhering L, Kupke A, Meier L, et al. Cyclophilin inhibitors restrict Middle East respiratory syndrome coronavirus via interferon- $\lambda$ in vitro and in mice. Eur Respir J 2020; 56: 1901826.

25 Carbajo-Lozoya J, Ma-Lauer Y, Malešević M, et al. Human coronavirus NL63 replication is cyclophilin A-dependent and inhibited by non-immunosuppressive cyclosporine A-derivatives including Alisporivir. Virus Res 2014; 184: 44-53. 
26 de Wilde AH, Zevenhoven-Dobbe JC, van de
coronaviruses. J Gen Virol 2011; 92: 2542-2548
27 Li HS, Kuok DIT, Cheung MC, et al. Effect

Li HS, Kuok DIT, Cheung MC, et al. Effect of interferon alpha and cyclosporine treatment separately and in combination on Middle East Respiratory Syndrome Coronavirus (MERS-CoV) replication in a human in-vitro and ex-vivo culture model. Antiviral Res 2018; 155: 89-96.

28 Hausenloy D, Boston-Griffiths E, Yellon D. Cyclosporin A and cardioprotection: from investigative tool to therapeutic agent. Br J Pharmacol 2012; 165: 1235-1245.

29 Liddicoat AM, Lavelle EC. Modulation of innate immunity by cyclosporine A. Biochem Pharmacol 2019; 163 : 472-480.

30 Elgebaly SA, Elbayoumi T, Kreutzer DL. Cyclosporin H: a novel anti-inflammatory therapy for influenza flu patients. J Egypt Soc Parasitol 2017; 47: 25-33.

31 Handschumacher RE, Harding MW, Rice J, et al. Cyclophilin: a specific cytosolic binding protein for cyclosporin A. Science 1984; 226: 544-547.

32 Friedman J, Weissman I. Two cytoplasmic candidates for immunophilin action are revealed by affinity for a new cyclophilin: one in the presence and one in the absence of CsA. Cell 1991; 66: 799-806.

33 Liu J, Farmer JD, Lane WS, et al. Calcineurin is a common target of cyclophilin-cyclosporin A and FKBP-FK506 complexes. Cell 1991; 66: 807-815.

$34 \mathrm{Li} \mathrm{YJ}, \mathrm{Wu} \mathrm{HH}$, Weng $\mathrm{CH}$, et al. Cyclophilin $\mathrm{A}$ and nuclear factor of activated $\mathrm{T}$ cells are essential in cyclosporine-mediated suppression of polyomavirus BK replication. Am J Transplant 2012; 12: 2348-2362.

35 Pfefferle S, Schöpf, J Kögl, M, et al. The SARS-coronavirus-host interactome: identification of cyclophilins as target for pan-coronavirus inhibitors. PLoS Pathog 2011; 7: e1002331.

36 De Wilde AH, Pham U, Posthuma CC, et al. Cyclophilins and cyclophilin inhibitors in nidovirus replication. Virology 2018; 522: 46-55

37 Tsuda K, Yamanaka K, Kitagawa H, et al. Calcineurin inhibitors suppress cytokine production from memory T cells and differentiation of naive T cells into cytokine-producing mature T cells. PLoS One 2012; 7: e31465.

38 Galani IE, Triantafyllia V, Eleminiadou EE, et al. Interferon-lambda mediates non-redundant front-line antiviral protection against influenza virus infection without compromising host fitness. Immunity 2017; 46: 875-890.

39 Bruening J, Weigel B, Gerold G. The role of type III interferons in hepatitis C virus infection and therapy. Immunol Res 2017; 2017: 1-12.

40 Behrens EM, Koretzky GA. Review: cytokine storm syndrome: looking toward the precision medicine era. Arthritis Rheumatol 2017; 69: 1135-1143.

41 Mehta P, McAuley DF, Brown M, et al. COVID-19: consider cytokine storm syndromes and immunosuppression. Lancet 2020; 395: 1033-1034.

42 Weaver LK, Behrens EM. Weathering the storm: improving therapeutic interventions for cytokine storm syndromes by targeting disease pathogenesis. Curr Treatm Opt Rheumatol 2017; 3: 33-48.

43 Campana C, Regazzi MB, Buggia I, et al. Clinically significant drug interactions with cyclosporin. An update. Clin Pharmacokinet 1996; 30: 141-179.

44 Iacono A, Wijesinha M, Rajagopal K, et al. A randomised single-centre trial of inhaled liposomal cyclosporine for bronchiolitis obliterans syndrome post-lung transplantation. ERJ Open Res 2019; 5: 00167-2019.

45 Neurohr C, Monforte V, Knoop C, et al. Aerosolized liposomal cyclosporine A in the prevention of bronchiolitis obliterans syndrome following lung transplantation. J Heart Lung Transplant 2015; 34: S243. 